Bull. Korean Math. Soc. 52 (2015), No. 2, pp. 619-626

http://dx.doi.org/10.4134/BKMS.2015.52.2.619

\title{
REGULARITY CRITERIA FOR THE $p$-HARMONIC AND OSTWALD-DE WAELE FLOWS
}

\author{
Jishan Fan, Gen Nakamura, And Yong Zhou
}

ABSTRACT. This paper considers regularity for the $p$-harmonic and Ostwald-de Waele flows. Some Serrin's type regularity criteria are established for $1<p<2$.

\section{Introduction}

In this paper, we consider the regularity criteria of the weak solutions of the $p$-harmonic flows:

$$
\begin{aligned}
& u_{t}-\operatorname{div}\left(|\nabla u|^{p-2} \nabla u\right)=u|\nabla u|^{p}, \\
& |u|=1, \\
& u(\cdot, 0)=u_{0},\left|u_{0}\right|=1, \text { in } \mathbb{R}^{n} .
\end{aligned}
$$

When $p=2$, it is the well-known harmonic heat flow, which has been widely studied $[5,6,7,11,19]$. The papers $[11,19]$ proved some regularity criteria.

When $p>n \geq 3$, Fardoun-Regbaoui [12] showed the global well-posedness of strong solutions for large data. Hungerbühler [14] established existence of global weak solutions of the $p$-harmonic flow between Riemannian manifolds $M$ and $N$ for arbitrary initial data having finite $p$-energy in the case when the target $N$ is a homogeneous space with a left invariant metric when $2<p<n$. Chen-Hong-Hungerbühler [8] proved existence of global weak solutions when $p \geq 2$.

When $1<p<2$, Misawa [18] proved that the problem (1.1)-(1.3) has a global weak solution satisfying

$$
\frac{1}{p} \int|\nabla u|^{p} d x+\int_{0}^{T} \int\left|u_{t}\right|^{2} d x d t \leq \frac{1}{p} \int\left|\nabla u_{0}\right|^{p} d x .
$$

Very recently, Iagar-Moll [15] studied the $p$-harmonic flow $(1<p<2)$ from the unit disk $D^{2}$ to the unit sphere $S^{2}$ under the rotational symmetry and they showed that the Dirichlet problem with constant boundary conditions

Received April 25, 2014; Revised September 17, 2014.

2010 Mathematics Subject Classification. 35K92, 76A15, 82D40.

Key words and phrases. $p$-harmonic flow, regularity criterion, weak solutions.

(C)2015 Korean Mathematical Society 
is locally well-posed in the class of classical solutions and they also gave a sufficient condition for the derivative of the solutions to blow-up in finite time.

The first aim of this paper is to prove some regularity criteria for the weak solutions of the problem (1.1)-(1.3) when $1<p<2$. We will prove:

Theorem 1.1. Let $n=3$ and $1<p<2$. Let $\nabla u_{0} \in L^{2} \cap L^{p}$ and $\left|u_{0}\right|=1$ in $\mathbb{R}^{n}$. Let $u$ be the weak solution constructed in [18]. If $\nabla u$ satisfies one of the following two conditions:

(i) $\nabla u \in L^{r}\left(0, T ; L^{s}\right)$ with $\frac{p}{r}+\frac{3}{s} \leq 1$,

$$
r=\frac{p\left(2 q-3+\frac{6}{p}\right)}{2 q-3}, s=\frac{q}{2} r, \frac{3}{2}<q \leq \infty,
$$

then we have$$
\text { (ii) } \nabla u \in L^{p}(0, T ; B M O) \text {, }
$$

Here BMO denotes the spaces of functions of bounded mean oscillations.

Remark 1.1. The system (1.1) has a scaling invariance under $u \rightarrow u_{\lambda}:=$ $u\left(\lambda x, \lambda^{p} t\right)$ for any $\lambda>0$. In this sense, the conditions (1.5) and (1.6) are optimal. We also point out that the paper [15] gave a special solution blowing up in finite time, while we here give a general blowing up condition.

Next, we consider the regularity of the weak solutions of the pseudo-plastic Ostwald-de Waele non-Newtonian models [2, 3]:

$$
\begin{aligned}
& \partial_{t} u_{i}+u \cdot \nabla u_{i}+\partial_{i} \pi-\sum_{j} \partial_{j} \Gamma_{i j}=0, \\
& \operatorname{div} u=0, \\
& \Gamma_{i j}:=|E(\nabla u)|^{p-2} E_{i j}(\nabla u), \\
& E_{i j}(\nabla u)=\frac{1}{2}\left(\partial_{j} u_{i}+\partial_{i} u_{j}\right), \quad i, j=1,2,3, \\
& u(\cdot, 0)=u_{0} \quad \text { in } \mathbb{R}^{3} .
\end{aligned}
$$

Here $u$ is the fluid velocity field and $\pi$ is the pressure.

Definition 1.1. Let $u_{0} \in L^{2}$ with $\operatorname{div} u_{0}=0$. We call $u \in L^{\infty}\left(0, T ; L^{2}\right) \cap$ $L^{p}\left(0, T ; W^{1, p}\right)$ a weak solutions of $(1.8)$ with bounded energy, if

$$
-\int_{0}^{T} \int u \phi_{t} d x d t-\int_{0}^{T} \int u \otimes u: \nabla \phi d x d t+\int_{0}^{T} \int|\nabla u|^{p-2} \nabla u: \nabla \phi d x d t=\int u_{0} \phi(0) d x
$$

for all $\phi \in C^{\infty}\left(\mathbb{T}^{3} \times[0, T]\right)$ with $\operatorname{div} \phi=0$ and there holds the following energy inequality

$$
\frac{1}{2}\|u(t)\|_{L^{2}}^{2}+\int_{0}^{t} \int|\nabla u|^{p} d x d s \leq \frac{1}{2}\left\|u_{0}\right\|_{L^{2}}^{2}
$$

for almost all $t \in(0, T)$. 
Definition 1.2. Let $u_{0} \in H^{1}$. We say that a weak solution $u$ is a strong solution to (1.8) if

$$
\begin{aligned}
& \nabla u \in L^{3}\left(\mathbb{R}^{3} \times(0, T)\right) \cap L^{\infty}\left(0, T ; L^{p} \cap L^{2}\right), \\
& u_{t} \in L^{2}\left(\mathbb{R}^{3} \times(0, T)\right),
\end{aligned}
$$

and there holds

$$
\int_{0}^{T} \int|\nabla u|^{p-2}\left|\nabla^{2} u\right|^{2} d x d t<\infty .
$$

The existence of weak solutions is shown in $[16,17]$ with the periodic boundary condition, and in [20] in the whole space, and in [10,21] in a bounded domain. The existence of strong solutions is proved in [16] for $p \geq \frac{11}{5}$ with the periodic boundary condition. For $\frac{9}{5}<p<2$, the existence of weak solutions of bipolar fluid is given in [17]. For $\frac{7}{5}<p<2$, the short time existence of strong solutions are obtained in $[4,9]$ with the periodic boundary condition. For $2<p$ and $\Omega:=\mathbb{T}^{3}$ or $\mathbb{R}^{3}$, the short time existence of strong solutions are proved in [3].

In [2], Bae-Choe-Kim proved the following regularity criterion

$u \in L^{\beta}\left(0, T ; L^{\alpha}\right)$ with $\frac{3}{\alpha}+\frac{5 p-6}{2 \beta} \leq \frac{5 p-8}{2} \quad\left(\frac{8}{5}<p<2\right)$ and $\frac{6}{5 p-8}<\alpha$.

Very recently, Bae-Kang-Lee-Wolf [3] showed the following regularity criterion:

$\nabla u \in L^{\beta}\left(0, T ; L^{\alpha}\right)$ with $\frac{3}{\alpha}+\frac{\frac{2}{3-p}}{\beta}=\frac{2}{3-p}\left(2<p<\frac{11}{5}\right)$ and $\frac{3(3-p)}{2}<\alpha$.

If we consider the scaling invariance for the system (1.8), the following scaling property of solutions is satisfied:

$$
\left(u_{\lambda}, \pi_{\lambda}\right):=\left(\lambda^{\frac{p-1}{3-p}} u\left(\lambda x, \lambda^{\frac{2}{3-p}} t\right), \lambda^{\frac{2 p-2}{3-p}} \pi\left(\lambda x, \lambda^{\frac{2}{3-p}} t\right)\right) .
$$

Therefore, a Serrin's type condition for $u$ is given as

$$
u \in L^{\beta}\left(0, T ; L^{\alpha}\right) \text { with } \frac{3}{\alpha}+\frac{\frac{2}{3-p}}{\beta}=\frac{p-1}{3-p} .
$$

In this sense, (1.12) is not optimal and (1.13) is optimal.

The second aim of this paper is to give more regularity criteria for the problem (1.8). We will prove:

Theorem 1.2. Let $\frac{7}{5}<p<2$ and $u_{0} \in H^{1}$ with $\operatorname{div} u_{0}=0$ in $\mathbb{R}^{3}$. If $\nabla u$ satisfies one of the following two conditions

$$
\text { (i) } \nabla u \in L^{\beta}\left(0, T ; L^{\alpha}\right) \text { with } \frac{3}{\alpha}+\frac{\frac{2}{3-p}}{\beta}=\frac{2}{3-p}
$$




$$
\text { and } \frac{3(3-p)}{2}<\alpha \leq \infty
$$

(ii) $\nabla u \in L^{1}(0, T ; B M O)$,

then we have

$$
u \in L^{\infty}\left(0, T ; H^{1}\right) \cap L^{p}\left(0, T ; W^{2, p}\right) .
$$

In the following proofs, we will use the following interpolation inequality $[13,1]$

$$
\|f\|_{L^{p}} \leq C\|f\|_{L^{q}}^{\frac{q}{p}}\|f\|_{B M O}^{1-\frac{q}{p}}
$$

with $1 \leq q<p<\infty$.

\section{Proof of Theorem 1.1}

This section is devoted to the proof of Theorem 1.1. We only need to establish (1.7) under (1.5) or (1.6) by formal calculations.

Testing (1.1) by $u_{t}$ and using $u \cdot u_{t}=0$, we see that (1.4) holds true.

Testing (1.1) by $-\Delta u$ and using $-u \cdot \Delta u=|\nabla u|^{2}$, we deduce that

$$
\frac{1}{2} \frac{d}{d t} \int|\nabla u|^{2} d x-\int|\nabla u|^{p-2} \sum_{i, j} \partial_{j} u_{i} \Delta \partial_{j} u_{i} d x=\int|\nabla u|^{p+2} d x .
$$

We estimate the second term of the left hand side as follows

$$
\begin{aligned}
I: & =-\sum_{i, j} \int|\nabla u|^{p-2} \partial_{j} u_{i} \Delta \partial_{j} u_{i} d x \\
& =\sum_{i, j} \int|\nabla u|^{p-2}\left|\nabla \partial_{j} u_{i}\right|^{2} d x+\sum_{i, j} \int \partial_{j} u_{i} \cdot \nabla \partial_{j} u_{i} \cdot \nabla|\nabla u|^{p-2} d x \\
& =\int|\nabla u|^{p-2}\left|\nabla^{2} u\right|^{2} d x+\frac{1}{2} \int \nabla|\nabla u|^{2} \cdot \nabla|\nabla u|^{p-2} \\
& \geq \int|\nabla u|^{p-2}\left|\nabla^{2} u\right|^{2} d x+\left.\left.C_{0} \int|\nabla| \nabla u\right|^{\frac{p}{2}}\right|^{2} d x
\end{aligned}
$$

Case 1. Let (1.5) hold true.

Letting $w:=|\nabla u|^{\frac{p}{2}}$, we estimate the right hand side of (2.1) as follows.

$$
\begin{aligned}
J: & =\int|\nabla u|^{p+2} d x \\
& \leq \int w^{2+\frac{4}{p}} d x=\int w^{\theta_{1}+\theta_{2}+\theta_{3}} d x \quad\left(\theta_{1}+\theta_{2}+\theta_{3}=2+\frac{4}{p}\right) \\
& \leq\left\|w^{\theta_{1}}\right\|_{L^{p_{1}}}\left\|w^{\theta_{2}}\right\|_{L^{p_{2}}}\left\|w^{\theta_{3}}\right\|_{L^{q}}\left(\frac{1}{p_{1}}+\frac{1}{p_{2}}+\frac{1}{q}=1\right) \\
& =\|w\|_{L^{\theta_{1} p_{1}}}^{\theta_{1}}\|w\|_{L^{\theta_{2} p_{2}}}^{\theta_{2}}\|w\|_{L^{\theta_{3} q}}^{\theta_{3}} \\
& \leq C\|w\|_{L^{6}}^{\theta_{1}}\|\nabla u\|_{L^{\frac{p}{2} \theta_{2} p_{2}}}^{\frac{p}{2} \theta_{2}}\|\nabla u\|_{L^{\frac{p}{2} \theta_{3} q}}^{\frac{p}{2} \theta_{3}}\left(\theta_{1} p_{1}=6\right)
\end{aligned}
$$




$$
\begin{aligned}
& \leq \frac{C_{0}}{2}\|\nabla w\|_{L^{2}}^{2}+C\left(\|\nabla u\|_{L^{\frac{p}{2} \theta_{2} p_{2}}}^{\frac{p}{2} \theta_{2}}\|\nabla u\|_{L^{s}}^{\frac{p}{2} \theta_{3}}\right)^{\frac{2}{2-\theta_{1}}} \\
& \left(p \theta_{2} p_{2}=4, \frac{p}{2} \theta_{2} \cdot \frac{2}{2-\theta_{1}}=2, \frac{p}{2} \theta_{3} q=s, \frac{p}{2} \theta_{3} \cdot \frac{2}{2-\theta_{1}}=r\right) \\
& \leq \frac{C_{0}}{2}\|\nabla w\|_{L^{2}}^{2}+C\|\nabla u\|_{L^{2}}^{2}\|\nabla u\|_{L^{s}}^{r},
\end{aligned}
$$

where we have used the following choice of the constants:

$$
\theta_{1}=\frac{3}{q}, p_{1}=2 q, \theta_{2}=\frac{2}{p}\left(2-\frac{3}{q}\right), p_{2}=\frac{4}{p \theta_{2}}, \theta_{3}=2-\frac{3}{q}+\frac{2}{p} \cdot \frac{3}{q} .
$$

Inserting the above estimates into (2.1) and using the Gronwall inequality, we obtain

$$
\int|\nabla u|^{2}+\int_{0}^{T} \int|\nabla u|^{p-2}\left|\nabla^{2} u\right|^{2} d x d t \leq C .
$$

On the other hand, it is easy to verity that

$$
\begin{aligned}
& \int_{0}^{T} \int\left|\nabla^{2} u\right|^{p} d x d t=\int_{0}^{T} \int|\nabla u|^{\frac{p(2-p)}{2}} \cdot|\nabla u|^{\frac{p(p-2)}{2}}\left|\nabla^{2} u\right|^{p} d x \\
\leq & \left(\int_{0}^{T} \int|\nabla u|^{p-2}\left|\nabla^{2} u\right|^{2} d x d t\right)^{\frac{p}{2}}\left(\int_{0}^{T} \int|\nabla u|^{p} d x d t\right)^{\frac{2-p}{2}} \\
\leq & \frac{p}{2} \int_{0}^{T} \int|\nabla u|^{p-2}\left|\nabla^{2} u\right|^{2} d x d t+\frac{2-p}{2} \int_{0}^{T} \int|\nabla u|^{p} d x d t \leq C .
\end{aligned}
$$

(2.4) and (2.5) imply (1.7).

This completes the proof of the case 1 .

Case 2. Let (1.6) hold true.

We still have (1.4), (2.1) and (2.2).

We use (1.18) to bound $J$ as follows.

$$
\begin{aligned}
J & \leq \int w^{2+\frac{4}{p}} d x \\
& \leq C \int|\nabla u|^{p+2} d x \\
& \leq C\|\nabla u\|_{L^{2}}^{2}\|\nabla u\|_{B M O}^{p} .
\end{aligned}
$$

Inserting (2.2) and (2.6) into (2.1) and using the Gronwall inequality, we have (2.4) and (2.5) and thus (1.7) holds true.

This completes the proof.

\section{Proof of Theorem 1.2}

This section is devoted to the proof of Theorem 1.2, like that in [2], we only prove the a priori estimates (1.17) under the condition (1.15) or (1.16) by formal calculations. 
First, we have the well-known energy inequality (1.10).

Testing (1.8) by $-\Delta u$ and using the divergence free property, we see that

$$
\begin{aligned}
& \frac{1}{2} \frac{d}{d t} \int|\nabla u|^{2} d x+\sum_{i, j} \int \partial_{j} \Gamma_{i j} \Delta u_{i} d x=\sum_{i, j} \int u_{j} \partial_{j} u_{i} \Delta u_{i} d x \\
= & -\sum_{i, j} \int \nabla u_{j} \partial_{j} u_{i} \nabla u_{i} d x \leq C \int|\nabla u|^{3} d x .
\end{aligned}
$$

By the same calculations as that in [2], we find that

$$
\begin{aligned}
& \partial_{k}\left(|E(\nabla u)|^{p-2} E_{i j}(\nabla u)\right) \partial_{k} E_{i j}(\nabla u) \\
= & \partial_{k}\left(\left(E_{l m}(\nabla u) E_{l m}(\nabla u)\right)^{\frac{p-2}{2}} E_{i j}(\nabla u)\right) \partial_{k} E_{i j}(\nabla u) \\
= & |E(\nabla u)|^{p-2} \partial_{k} E_{i j}(\nabla u) \partial_{k} E_{i j}(\nabla u) \\
& +(p-2)|E(\nabla u)|^{p-4} E_{l m}(\nabla u) \partial_{k} E_{l m}(\nabla u) E_{i j}(\nabla u) \partial_{k} E_{i j}(\nabla u) .
\end{aligned}
$$

Hence we obtain that

$\sum_{i, j} \int \partial_{j} \Gamma_{i j} \Delta u_{i} d x \geq C_{0} \int|E(\nabla u)|^{p-2}|\nabla E(\nabla u)|^{2} d x+\left.\left.C_{1} \int|\nabla| E(\nabla u)\right|^{\frac{p}{2}}\right|^{2} d x$.

Case 1. Let (1.16) hold true.

We use (1.18) to estimate the right hand side of (3.1) as follows.

$$
\int|\nabla u|^{3} d x \leq C\|\nabla u\|_{B M O}\|\nabla u\|_{L^{2}}^{2} .
$$

Inserting (3.4) and (3.3) into (3.1) and using the Gronwall inequality, we conclude that

$$
\int|\nabla u|^{2} d x+\int_{0}^{T} \int|E(\nabla u)|^{p-2}|\nabla E(\nabla u)|^{2} d x d t \leq C .
$$

Similarly to $(2.5)$, we have

$$
\int_{0}^{T} \int|\Delta u|^{p} d x d t \leq C
$$

This completes the proof of the case 1 .

Case 2. Let (1.15) hold true.

We denote $w:=|E(\nabla u)|^{\frac{p}{2}}$ and estimate the right hand side of (3.1) as follows.

$$
\begin{aligned}
\int|\nabla u|^{3} d x & \leq C \int w^{\frac{6}{p}} d x \\
& =C \int w^{\theta_{1}} \cdot w^{\theta_{2}} \cdot w^{\theta_{3}} d x \quad\left(\theta_{1}+\theta_{2}+\theta_{3}=\frac{6}{p}\right) \\
& \leq C\left\|w^{\theta_{1}}\right\|_{L^{p_{1}}}\left\|w^{\theta_{2}}\right\|_{L^{p_{2}}}\left\|w^{\theta_{3}}\right\|_{L^{q}} \quad\left(\frac{1}{p_{1}}+\frac{1}{p_{2}}+\frac{1}{q}=1\right)
\end{aligned}
$$




$$
\begin{aligned}
= & C\|w\|_{L^{\theta_{1} p_{1}}}^{\theta_{1}}\|\nabla u\|_{L^{\frac{p}{2} \theta_{2} p_{2}}}^{\frac{p}{2} \theta_{2}}\|\nabla u\|_{L^{\frac{p}{2} \theta_{3} q}}^{\frac{p}{2} \theta_{3}} \\
& \left(\theta_{1} p_{1}=6, \frac{p}{2} \theta_{2} p_{2}=2, \frac{p}{2} \theta_{3} q=\alpha\right) \\
\leq & \frac{C_{1}}{2}\|\nabla w\|_{L^{2}}^{2}+C\left(\|\nabla u\|_{L^{2}}^{\frac{p}{2} \theta_{2}}\|\nabla u\|_{L^{\alpha}}^{\frac{p}{2} \theta_{3}}\right)^{\frac{2}{2-\theta_{1}}} \\
& \left(\frac{p}{2} \theta_{2} \cdot \frac{2}{2-\theta_{1}}=2, \frac{p}{2} \theta_{3} \cdot \frac{2}{2-\theta_{1}}=\beta\right) \\
= & \frac{C_{1}}{2}\|\nabla w\|_{L^{2}}^{2}+C\|\nabla u\|_{L^{2}}^{2}\|\nabla u\|_{L^{\alpha}}^{\beta} .
\end{aligned}
$$

Here we have used the following choice of the constants

$$
\theta_{1}=\frac{3}{q}, p_{1}=2 q, \theta_{2}=\frac{2}{p}\left(2-\frac{3}{q}\right), p_{2}=\frac{4}{p \theta_{2}}, \theta_{3}=\frac{2}{p}-\frac{3}{q}+\frac{2}{p} \cdot \frac{3}{q} .
$$

Inserting (3.3) and (3.7) into (3.1) and using (1.10) and the Gronwall inequality, we arrive at (3.5) and (3.6).

This completes the proof.

Acknowledgment. The authors are indebted to the referee for careful reading of the paper and many nice suggestions and to Professor H.-O. Bae for sending them the interesting paper [2]. Fan is partially supported by NSFC (No. 11171154). This project is funded by the Deanship of Scientific Research (DSR), King Abdulaziz University, under Grant No. 27-130-35-HiCi. The authors, therefore acknowledge technical and financial support of KAU.

\section{References}

[1] J. Azzam and J. Bedrossian, Bounded mean oscillation and the uniqueness of active scalar equations, arXiv: 1108.2735 v2 [math. AP] 3 Nov 2012.

[2] H.-O. Bae, H. J. Choe, and D. W. Kim, Regularity and singularity of weak solutions to Ostwald-De Waele flows, J. Korean Math. Soc. 37 (2000), no. 6, 957-975.

[3] H.-O. Bae, K. Kang, J. Lee, and J. Wolf, Regularity for Ostwald-de Waele type shear thickening fluids, Nonlinear Differ. Equ. Appl. 2014(in press).

[4] L. C. Berselli, L. Diening, and M. Ruzicka, Existence of strong solutions for incompressible fluids with shear dependent viscosities, J. Math. Fluid Mech. 12 (2010), no. 1, $101-132$.

[5] M. Bertsch, R. dal Passo, and R. van der Hout, Nonuniqueness for the heat flow of harmonic maps on the disk, Arch. Ration. Mech. Anal. 161 (2002), no. 2, 93-112.

[6] M. Bertsch, R. dal Passo, and A. Pisante, Point singularities and nonuniqueness for the heat flow for harmonic maps, Comm. Partial Differential Equations 28 (2003), no. 5-6, $1135-1160$.

[7] K.-C. Chang, W.-Y. Ding, and R. Ye, Finite time blow-up of heat flow of harmonic maps from surface, J. Differential Geom. 36 (1992), no. 2, 507-515.

[8] Y. Chen, M-C. Hong, and N. Hungerbuhler, Heat flow of p-harmonic maps with values into spheres, Math. Z. 215 (1994), no. 1, 25-35.

[9] L. Diening and M. Ruzicka, Strong solutions for generalized Newtonian fluids, J. Math. Fluid Mech. 7 (2005), no. 3, 413-450. 
[10] L. Diening, M. Ruzicka, and J. Wolf, Existence of weak solutions for unsteady motions of generalized Newtonian fluid, Ann. Sc. Norm. Super. Pisa Cl. Sci. (5) 9 (2010), no. 1, $1-46$.

[11] J. Fan and T. Ozawa, Logarithmically improved regularity criteria for Navier-Stokes and related equations, Math. Methods Appl. Sci. 32 (2009), no. 17, 2309-2318.

[12] A. Fardoun and R. Regbaoui, Heat flow for p-harmonic maps with small initial data, Calc. Var. Partial Differential Equations 16 (2003), no. 1, 1-16.

[13] C. Fefferman and E. M. Stein, $H^{p}$ spaces of several variables, Acta Math. 129 (1972), no. 3-4, 137-193.

[14] N. Hungerbuhler, Global weak solutions of the p-harmonic flow into homogeneous space, Indiana Univ. Math. J. 45 (1996), no. 1, 275-288.

[15] R. G. Iagar and S. Moll, Rotationally symmetric p-harmonic flows from $D^{2}$ to $S^{2}$ : local well-posedness and finite time blow-up, arXiv:1305.6552v1[math. AP], 2013.

[16] O. A. Ladyzhenskaya, The Mathematical Theory of Viscous Incompressible Flow, 2nd edn. Gordon and Breach, New York, 1969.

[17] J. Málek, J. Nečas, M. Rokyta, and M. Ružička, Weak and Measure-valued Solutions to Evolutionary PDEs, Chapman \& Hall, 1996.

[18] M. Misawa, On the p-harmonic flow into spheres in the singular case, Nonlinear Anal. 50 (2002), no. 4, 485-494.

[19] T. Ogawa, Sharp Sobolev inequality of logarithmic type and the limiting regularity condition to the harmonic heat flow, SIAM J. Math. Anal. 34 (2003), no. 6, 1318-1330

[20] M. Pokorny, Cauchy problem for the non-Newtonian viscous incompressible fluid, Appl. Math. 41 (1996), no. 3, 169-201.

[21] J. Wolf, Existence of weak solutions to the equations of non-stationary motion of nonNewtonian fluids with shear rate dependent viscosity, J. Math. Fluid Mech. 9 (2007), no. $1,104-138$

JISHAN FAN

Department of Applied Mathematics

NANJING Forestry UNIVERSITY

NANJing 210037, P. R. CHINA

E-mail address: fanjishan@njfu.com.cn

Gen NAKAmura

DePARTMENT OF MATHEMATiCS

INHA UNIVERSITY

INCHEON 402-751, KOREA

E-mail address: nakamuragenn@gmail.com

YONG ZHOU

SCHOOL OF Mathematics

Shanghai University of Finance And Economics

Shanghai 200433, P. R. China

AND

Nonlinear Analysis and Applied Mathematics (NAAM) Research Group

King Abdulaziz University, Jeddah 21589, Saudi Arabia

E-mail address: yzhou@mail.shufe.edu.cn 